\title{
Allocation of visual attention in good and poor readers
}

\author{
JULIE R. BRANNAN and MARY C. WILLIAMS \\ University of New Orleans, New Orleans, Louisiana
}

\begin{abstract}
Twelve children, who were classified as good or poor readers, and 4 adults were given a task used to measure the ability to direct attention across visual space. Accuracy in detecting briefly presented target letters ( $\mathrm{S}$ or $\mathrm{N}$ ) was measured as a function of whether a cue did or did not correctly predict target location. Results showed that adults and good readers were able to direct attention effectively when given a cue that correctly predicted the location of the target letter, whereas poor readers were not. Poor readers also produced lower accuracy rates when the cue preceded the target by $100 \mathrm{msec}$ or less, but demonstrated equal accuracy when the asynchrony between cue and target was $150 \mathrm{msec}$ or more. Right-visual-field enhancement was found in adults and good readers, but not in poor readers. These results are discussed within the framework of current theories of reading disability.
\end{abstract}

Models of the reading process-arising from such diverse areas as psychology, education, and neurophysiology-are plentiful. Although a comprehensive review of reading research is not within the scope of this paper, we will describe an area of recent research that bears special relevance to the allocation of visual attention.

Lovegrove and his co-workers (Badcock \& Lovegrove, 1981; Lovegrove, Bowling, Badcock, \& Blackwood, 1980; Lovegrove \& Brown, 1978; Lovegrove, Heddle, \& Slaghuis, 1980; Lovegrove, Martin, \& Slaghuis, 1986) have studied a group of reading disabled children who also show differences in certain visual processes. In that they perform at average or above-average levels in subjects other than reading but score significantly below grade level in reading ability, these children differ from what have been termed "backward readers," who have difficulty in many areas of schoolwork. For this reason, these children have been called specific-reading-disabled (SRD). SRDs also differ from backward readers in other measures; for example, SRDs score at average or aboveaverage levels on intelligence quotient tests, whereas generally backward readers tend to score at lower levels. SRDs are also predominantly male (with a ratio of 3.3 to 1 ), in contrast to backward readers, who are approximately equal in number of males and females.

Lovegrove et al. (1986) describe three major aspects of visual processing in SRDs that differ from those of normal readers. First, SRDs have longer visual persistence durations at low spatial frequencies than do controls (Slaghuis \& Lovegrove, 1984). Second, SRDs are less sensitive to contrast at low spatial frequencies but equally

The authors would like to thank James G. May for many helpful suggestions and J. F. Noonan for computer programming. Requests for reprints should be sent to the first author, Department of Psychology, University of New Orleans, New Orleans, LA 70148. or more sensitive at high spatial frequencies (Martin \& Lovegrove, 1984). Third, SRDs are less sensitive than controls to all rates of flicker (Martin \& Lovegrove, 1984). These data suggest that there exists a subcategory of disabled readers who show deficits early in the visual processing hierarchy.

Williams and her co-workers have expanded upon Lovegrove's work by studying the perceptual consequences of a visual processing deficit in poor readers. Williams and Bologna (1985) demonstrated that poor readers show stronger perceptual grouping effects than good readers, as indexed by difficulty in selectively attending to the relevant portions of a figure (see Pomerantz \& Garner, 1973). This finding suggests that poor readers are more inclined toward transient, global processing. Using an object-superiority paradigm, Stevens and Williams (1986) found that with normal readers accuracy in detecting barely visible targets is strongly linked to the perceived depth of the surrounding context pattern. This is consistent with previous findings (Weisstein, Williams, \& Harris, 1982; Williams \& Weisstein, 1981, 1984). Poor readers, however, base their accuracy judgments on the perceived connectedness of the surrounding context. Since the perceptual distinction between connected and fragmented patterns has been linked to transient, global processing operations (Williams \& Weisstein, 1980), this also suggests a reliance on global processing in poor readers. Finally, May, Williams, and Dunlap (1986) discovered that poor readers require more time than either adults or good readers to make a judgment about the temporal order of two briefly presented stimuli. This result suggests that poor readers may have slow or sluggish processing capacities.

The perceptual processes involved in allocating attention to selected portions of the visual field have been studied extensively. Posner and his associates (Posner, Nissen, \& Ogden, 1978; Posner \& Snyder, 1974; Pos- 
ner, Snyder, \& Davidson, 1980) have developed a paradigm to study subjects' ability to direct attention to different points across the visual field. They have discovered that this ability is enhanced if a cue that gives information about the location of the target is presented foveally before the target presentation. These shifts of attention can occur along different points into the periphery without eye movements' taking place, indicating that attention is not strictly a foveal process. Eriksen and Hoffman (1972, 1974) and Shaw and Shaw (1977) have demonstrated similar benefits using cues presented outside the foveal field.

This investigation utilized Posner's paradigm to compare attentional shifts in children who were good and poor readers and in adults. Letters were used as stimuli in order to make the task more relevant to the actual process of reading. If poor readers have an attentional deficit, differences between this population and the other subject populations may be observed in accuracy, in the length of time between cue and target (stimulus onset asynchrony, or SOA) needed for optimal accuracy, and/or in a costbenefit analysis of cue information.

\section{METHOD}

\section{Subjects}

Four adults and 12 children, all with normal or corrected-tonormal vision, served as subjects. The adults, 2 males and 2 females, were graduate students at the University of New Orleans and had a mean age of 26 years, with a range of 22 to 33 . Three of the adult subjects were naive as to the purpose of the experiment; the 4th adult subject was one of the authors. Six of the children, 4 males and 2 females, had been classified as poor readers, having scored at least 1 year below grade level on the Diagnostic Reading Scales (Spache, 1981). Poor readers were participating in a reading clinic at the University of New Orleans, and had a mean age of 10.67 years. The remaining children, 4 males and 2 females, had been classified as good readers, having scored at least 1 year above grade level. The good readers were selected from local elementary schools, and had a mean age of 9.5 years. The children in the two groups were well matched in age, with the exception of one 13-year-old in the poor reader group.

\section{Stimuli and Apparatus}

The stimuli, which consisted of green letters on a black background, were presented by a microcomputer (Zenith Z-100). The screen was covered by opaque black cardboard to eliminate reflection from room light. In the center of the cardboard was a viewing square covered by a $75 \times 75 \mathrm{~mm} 1.0$ neutral density filter (Kodak Wratten gelatin filter).

The targets were the letters $S$ and $N$, which appeared $2^{\circ}$ to the right or to the left of a fixation cross in the center of the viewing square. The targets subtended $.5^{\circ}$. A vertical line was presented as a cue to target presentation, either simultaneously with or at a variable time (SOA) before the onset of the target letter. The cue appeared $2^{\circ}$ into the periphery, $.8^{\circ}$ above the position where the target letter appeared. The SOA between the cue and target varied, with values of $0,50,100,150$, and $170 \mathrm{msec}$. The cue and target were presented for exactly $30 \mathrm{msec}$ each. In the longest SOA, the total time for one trial $(230 \mathrm{msec})$ still should have precluded any anticipatory eye movements (Breitmeyer, 1983; Breitmeyer \& Ganz, 1976).
A cost-benefit paradigm was utilized, with two probability conditions. In one condition, the probability that the position of the cue would accurately predict the position where the target would appear (left or right of fixation) was $50 \%$ (random). In the other condition, the probability that the cue would predict the position of the target was $80 \%$. The order in which these two conditions were presented was randomized among subjects to offset any fatigue or practice effects that might occur.

\section{Procedure}

A subject was seated in a semidark room at a viewing distance of $40 \mathrm{~cm}$ from a fixation cross in the center of the viewing square. The experimental procedure was explained clearly, with visible examples being included. The testing did not begin until the subject had demonstrated complete understanding of the procedure. The subject was asked to detect the letters $\mathrm{S}$ and $\mathrm{N}$ while fixating the viewing cross. A practice run of 10 trials at the $50 \%$ condition was conducted for each subject before the experimental session was begun. Each subject produced between $75 \%$ and $80 \%$ accuracy as a baseline measure.

Within each experimental session, the probability conditions $(50 \%$ or $80 \%$ ) were run blocked. Within both blocks, SOA and target alternative (S or $N$ ) were randomized, with 16 trials at each SOA. For each SOA, eight of each target alternative appeared, four on the left and four on the right of the fixation cross. A total of $\mathbf{8 0}$ trials were run for each of the two probability conditions. Each experimental session required less than $1 \mathrm{~h}$ of time.

\section{RESULTS}

A four-way analysis of variance (subject group $\times$ probability condition $\times$ SOA $\times$ cue prediction) was performed on the accuracy data. The main effect for groups (good readers, poor readers, and adults) was not significant. This is likely due to the fact that averaging across experimental variables obscures group differences.

The main effect for probability condition $(50 \%$ vs. $80 \%$ ) was significant $[F(1,13)=6.95, p<.01]$, suggesting that the percentage of time that the cue correctly predicted target location affected accuracy in target detection.

The main effects for SOA $[F(4,52)=7.33, p<.001]$ and validity of cue prediction $[F(1,13)=10.08$, $p<.001]$ were also significant, suggesting that the amount of time between cue and target (SOA), as well as whether or not the cue correctly predicted target location, significantly affected accuracy rates.

The interaction between groups and probability condition was significant $[F(2,13)=4.51, p<.05]$, showing that the effect of probability condition ( $50 \%$ vs. $80 \%)$ varied across subject populations. There were large differences in accuracy between the $50 \%$ and $80 \%$ conditions for good readers and adults (Newman-Keuls multiple comparison tests, $p<.05$ ) but not for poor readers (Newman-Keuls multiple comparison tests, $p>.05$ ). There were no significant differences between groups for the $50 \%$ condition, but poor readers produced significantly lower means (no benefit) on the $80 \%$ condition than either good readers or adults (Newman-Keuls multiple comparison tests, $p<.05$ ). This indicates that adults and 
good readers utilize cue information, whereas poor readers do not.

A second finding was that the groups differed in their ability to utilize cue information. Consider the predictive validity of the cue on each trial. Within each probability condition, the cue would correctly predict the target on some trials, but not on others. The interaction of subject group $\times$ probability condition $\times$ cue prediction was significant $[F(2,13) \neq 7.84, p<.01]$. The relationship between accuracy in target detection and the predictive validity of the cue is shown in Figure 1 for adults (top panel), good readers (middle panel), and poor readers (bottom panel). For good readers and adults, cue prediction had no effect on accuracy in the $50 \%$ condition (NewmanKeuls multiple comparison tests, $p>.05$ ), but led to an increase in accuracy when the cue correctly predicted the position of the target in the $80 \%$ condition (NewmanKeuls multiple comparison tests, $p<.05$ ). This indicates that adults and good readers are able to use information about target location more often when the cue correctly predicts target location than when it does not. For poor readers, cue prediction did not affect accuracy in either the $50 \%$ or the $80 \%$ condition (Newman-Keuls multiple comparison tests, $p>.05$ ).

The results of this interaction clearly demonstrate that the groups differed in their ability to utilize a cue to direct visual attention. Good readers and adults showed an enhancement of accuracy in the $80 \%$ condition when the cue correctly predicted target location. This constitutes the benefit in cost-benefit analysis. There was, however, a decrease in accuracy for the adults in the $80 \%$ condition when the cue did not accurately predict target location. This constitutes the cost in cost-benefit analysis. Poor readers, on the other hand, showed no accuracy differences for the $50 \%$ and the $80 \%$ conditions. Accuracy was not affected by whether the cue correctly or incorrectly predicted target location; thus, there are neither costs nor benefits on detection accuracy. This suggests that the information provided by the cue was not being utilized by poor readers.

A third finding in this study was that the groups differed in accuracy across SOAs. The interaction between subject group and accuracy at each SOA was significant $[F(8,52)=11.91, p<.001]$, showing that the effect of SOA (the time delay between the cue and target presentation) differed across subject groups. The relationship of target detection and SOA between cue and target is presented in Figure 2 for the three subject groups. Post hoc tests revealed that all three groups generated essentially flat functions. Within each subject group, accuracy did not significantly differ as a function of SOA (Newman-Keuls multiple comparison tests, $p>.05$ ). There were, however, some differences in accuracy between subject groups. For the shorter SOAs (0 and $50 \mathrm{msec}$ ), poor readers demonstrated significantly lower accuracy rates than good readers or adults. This difference disappears at the three longest SOAs (Newman-Keuls multiple comparison tests, $p<.05$ ). This suggests that poor readers require more time to shift visual attention
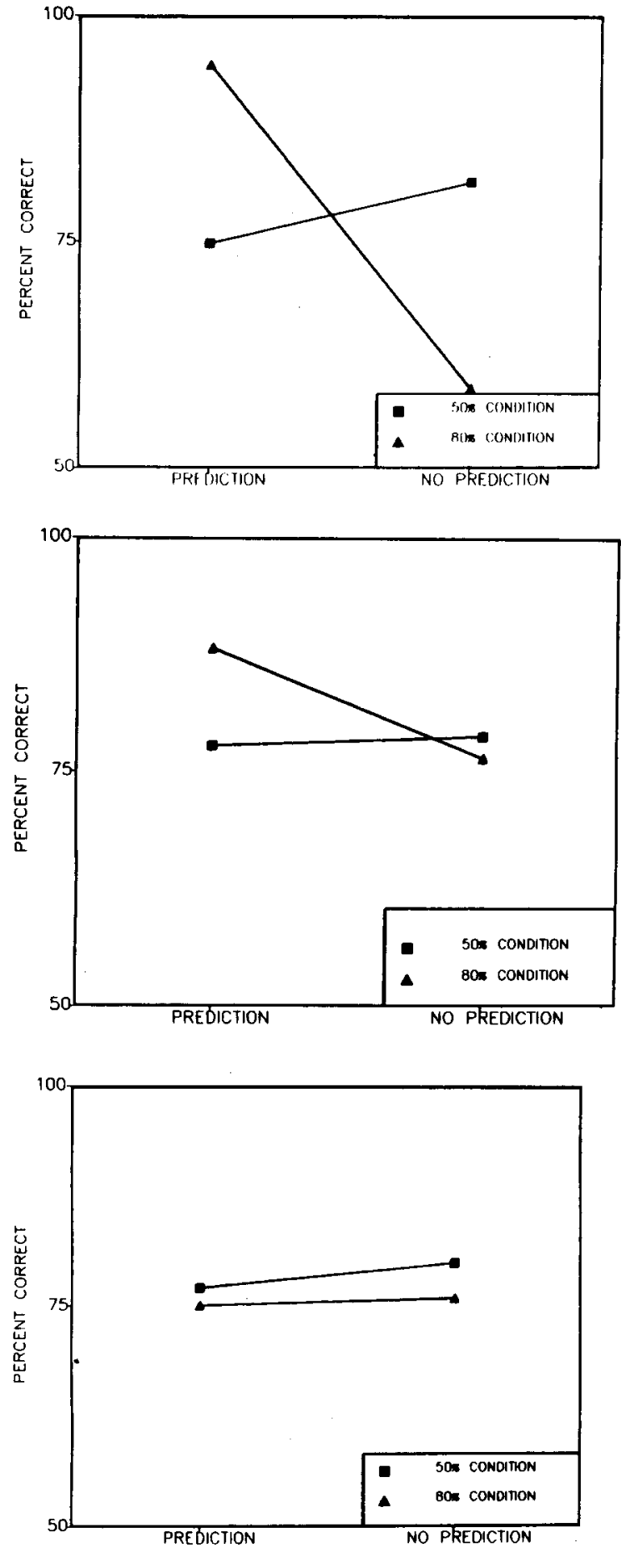

CUE PREDICTION

Figure 1. Cost-benefit effect in adult (top panel), good reader (middle panel), and poor reader (bottom panel) subject data. Accuracy is plotted as a function of whether or not the cue correctly predicted target location. Correct prediction occurred on $50 \%$ of the trials (squares) or $80 \%$ of the trials (triangles). The difference between the $50 \%$ and $80 \%$ conditions when the cue predicted constitutes the accuracy benefit; the same difference when the cue did not predict constitutes the accuracy cost. Adults show a pronounced cost-benefit effect, good readers show a lesser effect, and poor readers show no costs or benefits. 


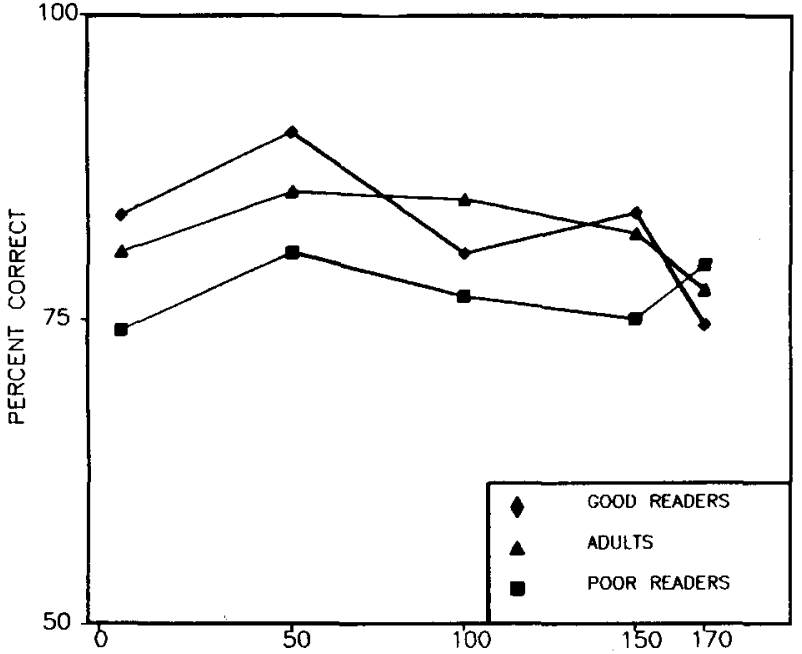

Figure 2. Accuracy is plotted as a function of stimulus onset asynchrony (SOA) for adults (triangles), good readers (diamonds), and poor readers (squares).

than good readers and adults at shorter temporal intervals but not at longer intervals.

The final finding of this study was that target position (target appearing to the left or to the right of the fixation cross) affected accuracy rates in good readers and adults but not in poor readers. A two-way ANOVA (subject group $X$ position) revealed a significant main effect of target position $[F(1,13)=114.5, p<.001]$ and a significant interaction between groups and position $[F(2,13)=34.65$, $p<.001]$. Accuracy in target detection when the target appeared on the left versus the right of the fixation cross is shown in Figure 3 for the three subject groups. Good readers and adults produced significantly higher accuracy rates when the target appeared on the right versus the left

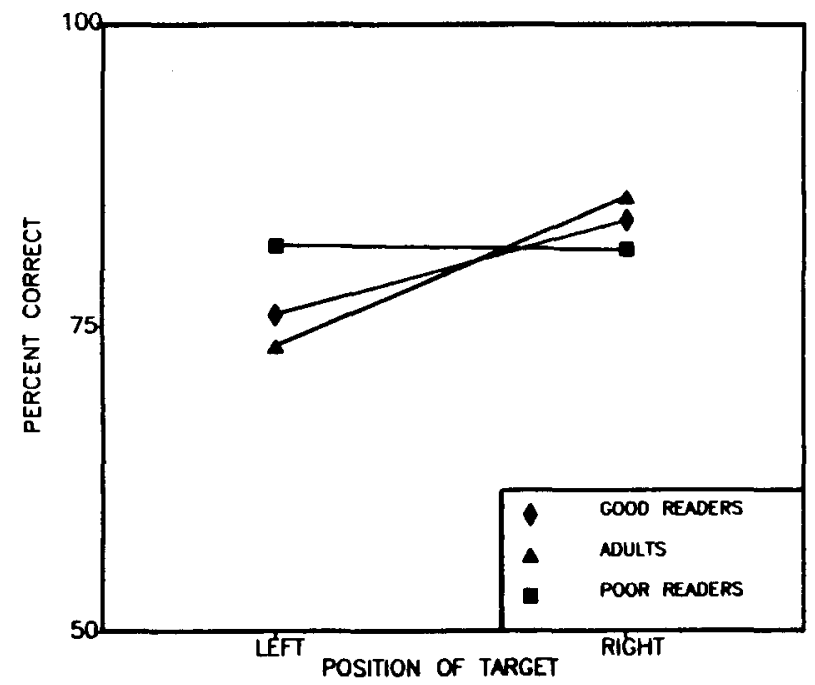

Figure 3. Accuracy is plotted as a function of whether the target appeared to the left or the right of the fixation cross for adults (triangles), good readers (diamonds), and poor readers (squares). of the fixation cross; poor readers showed no such effect (Newman-Keuls multiple comparison tests, $p<.05$ ).

A two-way ANOVA (subject group $\times$ target alternative) failed to reveal a significant main effect for accuracy in detection of the two target alternatives ( $\mathrm{S}$ vs. $\mathrm{N}$ ), with mean accuracy for $S$ and $N$ being 79.57 and 80.28 , respectively. Similarly, the interaction between groups and target alternative was not significant. These results suggest that the effects found in this experiment were not affected by which target alternative appeared on any given trial, supporting the validity of using pooled data from both target alternatives.

\section{DISCUSSION}

The main finding of this study is that children scoring at least 1 year below grade level in reading ability (poor readers) differ significantly both from children who are good readers and from adults in performance on a visual attention task. Good readers and adults are able to allocate attention across different points in visual space without making eye movements. Unlike good readers and adults, poor readers do not utilize location information provided by parafoveally presented cues to report which of two letters is also appearing parafoveally. The performance of good readers, adults, and poor readers is equivalent when cues do not provide information about the location of the target letter ( $50 \%$ condition), but when cues do often provide accurate information about the location (80\% condition), good readers and adults show an enhancement of accuracy whereas poor readers do not. This increase in accuracy in good readers and adults is a measure of their ability to allocate attention.

The performance of adults and good readers parallels Posner et al.'s (1978) descriptions of results obtained using a similar paradigm with adults. Unlike good readers and adults, the poor readers in the present study did not perform according to the cost-benefit rule. They produced the same results on both the $50 \%$ and the $80 \%$ conditions. In light of Posner et al.'s contention that the benefits seen in a situation like the present study's $80 \%$ condition were due to active attention, this result tends to suggest an attentional deficit of some kind in poor readers.

This attentional deficit could arise in various ways. Lovegrove et al. (1986) have suggested that the visual processing deficits found in SRDs could be the result of a transient system deficit. This explanation is based upon the reading model proposed by Breitmeyer $(1980,1983)$, and is an extension of an earlier model of visual masking processes (Breitmeyer \& Ganz, 1976). As described in this earlier work, the transient system responds briefly to abrupt on- and offsets and rapid motion. In contrast, the sustained system prefers slowly moving or stationary stimuli and produces a more tonic response. The model is based on the premise that fixation-saccade sequences in reading are mediated by the interaction of these transient and sustained channels in the visual system. Specifically, transient activity generated by saccades functions 
to inhibit the long-persisting sustained response elicited by each fixation, thus creating discrete and unmasked fixation intervals. According to this model, then, reading behavior would be influenced by the integrity of these channels.

Breitmeyer and Ganz (1976) state that they "believe that specifically it is transient neurons ... which direct the selective attention mechanisms"' (p. 28). They then provide three pieces of evidence to support this contention. First, in metacontrast studies (e.g., Fehrer \& Raab, 1962), subjects can locate a target quickly even if the target's shape is suppressed. This suggests that a rapidly responding (i.e., transient) channel is carrying location information. Second, it has been suggested that the amount of time required to switch attention psychophysically (LaBerge, 1973) is roughly equal to the $50-100 \mathrm{msec}$ interval between the rapid transient activity and slower sustained activity reaching the cortex (Breitmeyer \& Ganz, 1976). Third, attention to a flashed display is drawn first to the peripheral areas, where the concentration of transient cells is high (Hoffman, Stone, \& Sherman, 1972), and then moves toward the center, where the concentration of sustained cells is high.

If there is a transient deficit in these poor readers, it might result in this inability to utilize cue information that appears suddenly in the parafovea or periphery. An alternative explanation could describe the ability to use attentional resources flexibly as a skill learned and perfected by reading often. As shown in Figure 1, the adults demonstrated a more pronounced cost-benefit effect than did the good readers. Here, as reading improved, proficiency in manipulating attentional resources would develop as well. A feasible explanation would be an interactive one, involving both skill level and transient processing characteristics.

A second finding in this study is that the temporal responses shown by the poor readers were different from those shown by the good readers and adults. As shown in Figure 2, at shorter SOAs, the poor readers were less accurate than the good readers or adults. Accuracy differences disappear beyond $50 \mathrm{msec}$. This may be related to May et al.'s (1986) finding that poor readers require a significantly longer SOA than good readers to make a correct judgment about which of two word stimuli appears first. Both studies suggest that poor readers require more time to detect the temporal ordering of events. Given that the poor readers in the present study showed accuracy increases with increasing SOA, it is possible that poor readers are unable to utilize cue information because they cannot detect the temporal ordering of cue and target. It thus may not be that poor readers are unable to efficiently shift attention, but that they require a longer time to do so.

Finally, these data demonstrate a difference between poor readers and the other two groups when the target appears in the right visual field. Good readers and adults were significantly more accurate when the target appeared on the right side of the fixation cross, but poor readers were equally accurate for either side. It may be that skilled or practiced readers (i.e., adults and good readers) develop an automatic bias toward information processing in the right visual field but that less skilled or practiced readers (poor readers) have failed to develop this bias. Further studies should perhaps be conducted to explore this difference.

In conclusion, using a paradigm similar to Posner et al.'s (1978), this investigation revealed an apparent attentional deficit in children who read poorly. Planned future investigations in this area include replicating this procedure using foveal instead of peripheral cues in order to determine whether the effects seen in this study are due to peripheral processing deficits or to a lack of ability to use cue information in general.

\section{REFERENCES}

BADCock, D., Lovegrove, W. (1981). The effects of contrast, stimulus duration, and spatial frequency on visible persistence in normal and specifically disabled readers. Journal of Experimental Psychology: Human Perception \& Performance, 7, 495-505.

BREITMEYER, B. (1980). Unmasking visual masking: A look at the "why" behind the veil of "how." Psychological Review, 87, 52-69.

BREITMEYER, B. (1983). Sensory masking, persistence, and enhancement in visual exploration and reading. In K. Raynor (Ed.), Eye movements in reading. London: Academic Press.

BreITMEYER, B. G., \& GANZ, L. (1976). Implications of sustained and transient channels for theories of visual pattern masking, saccadic suppression, and information processing. Psychological Review, 83, 1-36.

ERIKSEN, C. W., \& HofFMAN, J. E. (1972). Temporal and spatial characteristics of selective encoding from visual displays. Perception \& Psychophysics, 12, 201-204.

ERIKSEN, C. W., \& Hoffman, J. E. (1974). Selective attention: Noise suppression or signal enhancement? Bulletin of the Psychonomic Society, 4, 587-589.

Fehrer, E., \& RAAB, D. (1962). Reaction time to stimuli marked by metacontrast. Journal of Experimental Psychology, 63, 143-147.

Hoffman, K.-P., Stone, J., \& Sherman, S. M. (1972). Relay of receptive-field properties in dorsal lateral geniculate nucleus of the cat. Journal of Neurophysiology, 35, 518-531.

LABERGE, D. (1973). Identification of the time to switch attention: A test of a serial and parallel model of attention. In S. Kornblum (Ed.), Attention and performance $I V$. New York: Academic Press.

Lovegrove, W., Bowling, A., BADCock, D., Blackwood, M. (1980). Specific reading disability: Differences in contrast sensitivity as a function of spatial frequency. Science, 210, 439-440.

Lovegrove, W., Brown, C. (1978). Development of information processing in normal and disabled readers. Perceptual \& Motor Skills, 46, 1047-1054

Lovegrove, W. J., Heddle, M., \& Slaghuis, W. (1980). Reading disability: Spatial frequency specific deficits in visual information store. Neuropsychologia, 18, 111-115.

Lovegrove, W., Martin, F., Slaghuis, W. (1986). A theoretical and experimental case for a visual deficit in specific reading disability. Cognitive Neuropsychology, 3, 225-267.

Martin, F., Lovegrove, W. (1984). The effects of field size and luminance on contrast sensitivity differences between specifically reading disabled and normal children. Neuropsychologia, 22, 73-77.

MAY, J., Willams, M., \& DuNLAP, W. (1986). Temporal order judgements in good and poor readers. Manuscript submitted for publication.

Pomerantz, J., \& GARner, W. (1973). Stimulus configuration in selective attention tasks. Perception \& Psychophysics, 14, 565-569.

Posner, M. I., Nissen, M. J., \& OGDen, W. C. (1978). Attended and unattended processing modes: The role of set for spatial location. In 
H. J. Pick \& I. J. Saltzman (Eds.), Modes of perception. Hillsdale, NJ: Erlbaum.

PosNer, M. I., \& SNYDER, C. R. (1974). Attention and cognitive control. In R. L. Solso (Ed.), Information processing and cognition: The Loyola Symposium. Hillsdale, NJ: Erlbaum.

Posner, M. I., SNyder, C. R. R., \& Davidson, B. J. (1980). Attention and the detection of signals. Journal of Experimental Psychology: General, 109, 160-174.

Shaw, M. L., \& SHaw, P. (1977). Optimal allocation of cognitive resources to spatial locations. Journal of Experimental Psychology: Human Perception \& Performance, 3, 201-211.

Slaghuis, W., \& LoVEgrove, W. J. (1984). Flicker masking of spatial frequency dependent visual persistence and specific reading disability. Perception, 13, 527-534.

SPACHE, G. (1981). Diagnostic Reading Scales. Monterey, CA: McGraw-Hill.

Stevens, P., \& Williams, M. (1986). The object-superiority effect in good and poor readers. Manuscript submitted for publication.
Weisstein, N., Williams, M. C., \& Harris, C. (1982). Depth, connectedness, and structural relevance in the object-superiority effect: Line segments are harder to see in flatter patterns. Perception, 11, 5-17. Williams, M., \& Bologna, N. (1985). Perceptual grouping effects in good and poor readers. Perception \& Psychophysics, 38, 367-374. Williams, M., \& Weisstein, N. (1980). Perceptual grouping produces spatial-frequency specific effects on metacontrast. Investigative Ophthalmology \& Visual Science, 21, 165. (Abstract)

Williams, M., \& Weisstein, N. (1981). Spatial frequency response and perceived depth in the time-course of object-superiority. Vision Research, 21, 631-646.

Williams, M., \& WeissteIN, N. (1984). The effect of perceived depth and connectiveness on metacontrast functions. Vision Research, 24, $1279-1288$

(Manuscript received March 25, 1986; revision accepted for publication September 23, 1986.) 